\title{
Contribution of gut bacteria to the metabolism of cyanidin 3-glucoside in human microbiota-associated rats
}

\author{
Laura Hanske, Wolfram Engst, Gunnar Loh, Silke Sczesny, Michael Blaut and Annett Braune* \\ Department of Gastrointestinal Microbiology, Analytics Group, German Institute of Human Nutrition Potsdam-Rebbruecke, \\ Arthur-Scheunert-Allee 114-116, 14558 Nuthetal, Germany
}

(Submitted 2 April 2012 - Final revision received 22 June 2012 - Accepted 3 July 2012 - First published online 21 August 2012)

\section{Abstract}

Cyanidin 3-glucoside (C3G) is one of the major dietary anthocyanins implicated in the prevention of chronic diseases. To evaluate the impact of human intestinal bacteria on the fate of $\mathrm{C} 3 \mathrm{G}$ in the host, we studied the metabolism of C3G in human microbiota-associated (HMA) rats in comparison with germ-free (GF) rats. Urine and faeces of the rats were analysed for C3G and its metabolites within $48 \mathrm{~h}$ after the application of $92 \mu \mathrm{mol} \mathrm{C} 3 \mathrm{G} / \mathrm{kg}$ body weight. In addition, we tested the microbial C3G conversion in vitro by incubating C3G with human faecal slurries and selected human gut bacteria. The HMA rats excreted with faeces a three times higher percentage of unconjugated C3G products and a two times higher percentage of conjugated C3G products than the GF rats. These differences were mainly due to the increased excretion of 3,4-dihydroxybenzoic acid, 2,4,6-trihydroxybenzaldehyde and 2,4,6-trihydroxybenzoic acid. Only the urine of HMA rats contained peonidin and 3-hydroxycinnamic acid and the percentage of conjugated C3G products in the urine was decreased compared with the GF rats. Overall, the presence of intestinal microbiota resulted in a $3.7 \%$ recovery of the C3G dose in HMA rats compared with $1.7 \%$ in GF rats. Human intestinal bacteria rapidly degraded C3G in vitro. Most of the C3G products were also found in the absence of bacteria, but at considerably lower levels. The higher concentrations of phenolic acids observed in the presence of intestinal bacteria may contribute to the proposed beneficial health effects of C3G.

Key words: Cyanidin 3-glucoside: Human microbiota-associated rats: Bacterial conversion: Human intestinal microbiota

Anthocyanins are abundant flavonoids in deep-coloured fruits and vegetables ${ }^{(1,2)}$. They have received considerable attention due to their potential health effects. The consumption of anthocyanin-rich foods is associated with a lower risk of all-cause mortality and death due to $\mathrm{CHD}$ and $\mathrm{CVD}^{(3)}$. Also, experimental studies have indicated protection by anthocyanins against other age-related disorders, such as cancer and neurodegenerative diseases $^{(4-6)}$. The daily anthocyanin intake was estimated to range between $2.7 \mathrm{mg}$ in Germany and $82.5 \mathrm{mg}$ in $\operatorname{Finland}^{(7,8)}$, with cyanidin glycosides accounting for $44.7 \%$ of total intake $^{(1)}$. Cyanidin 3-glucoside (C3G) is one of the most common anthocyanins contained in dietary plants such as berries $^{(9)}$. In contrast to other flavonoids, anthocyanins are absorbed as intact glycosides ${ }^{(9)}$. However, the systemic availability of anthocyanins, their aglycones and the corresponding conjugates is poor. Less than $1 \%$ of the ingested anthocyanins are typically absorbed and excreted in the urine ${ }^{(10)}$. Since the concentration of the parent anthocyanins in the body is low, it has been proposed that their derived metabolites mediate the biological activities and the ensuing health effects of anthocyanins, either directly in the intestine or after absorption ${ }^{(11)}$. A substantial proportion of ingested anthocyanins enters the large intestine ${ }^{(12)}$ and, thus, gut bacteria affect the fate of anthocyanins. Intestinal microbiota from humans and laboratory animals extensively convert anthocyanins in vitro ${ }^{(13-17)}$. Moreover, anthocyanins undergo rearrangements in response to $\mathrm{pH}$. At neutral $\mathrm{pH}$, in particular, anthocyanin aglycones are spontaneously degraded to their corresponding phenolic acids and aldehydes by cleavage of the $\mathrm{C}$-ring ${ }^{(2,18)}$. Most studies on the metabolism of $\mathrm{C} 3 \mathrm{G}$ in vitro and in vivo have revealed 3,4-dihydroxybenzoic acid (DHBA, protocatechuic acid) as one of the major degradation products ${ }^{(13,19-21)}$. So far, the influence of human intestinal bacteria on the metabolism of C3G and other anthocyanins in vivo is not yet clear.

In the present study, we used rats associated with a human intestinal microbiota (human microbiota-associated (HMA) rats) to investigate the impact of human gut bacteria on the metabolism of $\mathrm{C} 3 \mathrm{G}$. The metabolic fate of $\mathrm{C} 3 \mathrm{G}$ in HMA rats

Abbreviations: AUC, area under the curve; C3G, cyanidin 3-glucoside; DHBA, 3,4-dihydroxybenzoic acid; GF, germ-free; HCA, 3-hydroxycinnamic acid; HMA, human microbiota-associated; TFA, trifluoroacetic acid; THBA, 2,4,6-trihydroxybenzoic acid; THBAld, 2,4,6-trihydroxybenzaldehyde; UPLC, ultraperformance liquid chromatography; VA, vanillic acid.

*Corresponding author: Dr A. Braune, fax + 4933200 882407, email braune@dife.de 
was compared with that of germ-free (GF) rats by analysing urine and faeces. In vitro investigations on the degradation of $\mathrm{C} 3 \mathrm{G}$ in the absence and presence of human intestinal bacteria were performed in parallel.

\section{Materials and methods}

\section{Chemicals}

C3G (purity, 98\%) isolated from blackberries by countercurrent chromatography ${ }^{(22)}$ was provided by Peter Winterhalter (Institute of Food Chemistry, Technical University of Braunschweig). Cyanidin, peonidin, hippuric acid and phloroglucinol were purchased from Roth. 3-Hydroxyphenylacetic acid, 2-hydroxybenzoic acid, 3-hydroxybenzoic acid, 4-hydroxybenzoic acid, benzoic acid, 3-hydroxycinnamic acid (HCA), ferulic acid, vanillic acid (VA, 4-hydroxy-3-methoxybenzoic acid), 3-methoxybenzoic acid, 2,4,6-trihydroxybenzaldehyde (THBAld), 2,4,6-trihydroxybenzoic acid (THBA), DHBA, 2,4-dihydroxybenzoic acid, 2,6-dihydroxybenzoic acid and catechol were obtained from Sigma-Aldrich. 3-Phenylpropionic acid, 3,4-dihydroxycinnamic acid and phenylacetic acid were purchased from Acros. 3-(3-Hydroxyphenyl)propionic acid was from Alfa Aesar.

\section{Degradation experiments with human intestinal bacteria}

Faecal suspensions were prepared in an anoxic workstation (MAKS MG, Meintrup dw scientific) with a gas phase of $\mathrm{N}_{2}-\mathrm{CO}_{2}-\mathrm{H}_{2}$ (80:10:10, by vol.). Human faeces from one male and three female healthy volunteers, aged 23-45 years and consuming an unspecified Western diet, who had not taken antibiotics for at least 6 months before the study, were each diluted to $1 \%(\mathrm{w} / \mathrm{v})$ in carbonate-phosphate buffer (0.006 $\mathrm{m}$-carbonate and 0.095 $\mathrm{m}$-phosphate, $\left.\mathrm{pH} 5 \cdot 5^{(13)}\right)$.

For incubation experiments with Clostridium saccharogumia DSM 17460 and Eubacterium ramulus DSM 16296 (German Collection of Microorganisms and Cell Cultures), the bacteria were grown in a complex medium (standard (ST) medium $\left.{ }^{(23)}\right)$. The cells were harvested $\left(8000 \mathrm{~g}, 3 \mathrm{~min}, 4^{\circ} \mathrm{C}\right)$, washed with carbonate-phosphate buffer and resuspended in the same buffer at concentrations of $1.7 \times 10^{8}$ cells $/ \mathrm{ml}$ for C. saccharogumia and $1 \times 10^{9}$ cells $/ \mathrm{ml}$ for E. ramulus.

A $100 \mu \mathrm{l}$ stock solution of $10 \mathrm{~mm}-\mathrm{C} 3 \mathrm{G}$ in methanol was added to $9.9 \mathrm{ml}$ of bacterial suspensions. Cultures were incubated under a gas phase of $\mathrm{N}_{2}-\mathrm{CO}_{2}(80: 20, \mathrm{v} / \mathrm{v})$ in $16 \mathrm{ml}$ Hungate tubes, fitted with butyl rubber stoppers and screw caps, at $37^{\circ} \mathrm{C}$ in a water-bath equipped with a rotary shaker. $\mathrm{C} 3 \mathrm{G}$ and bacterial suspensions incubated separately in medium served as controls. Aliquots were taken at different time points, acidified with one volume of $1 \%$ aqueous trifluoroacetic acid (TFA, v/v) and immediately lyophilised. All incubations were carried out in triplicate.

\section{Incubation in simulated gastric and intestinal fluids}

C3G and cyanidin were each incubated with simulated gastric fluid or simulated intestinal fluid under continuous shaking at $37^{\circ} \mathrm{C}$ as described previously ${ }^{(24)}$. The compounds were added from $10 \mathrm{~mm}$ stock solutions in methanol to a final concentration of $100 \mu \mathrm{m}$ each. Aliquots were taken after 15, 30, 60, 90 and $120 \mathrm{~min}$ of incubation, lyophilised and analysed by HPLC.

\section{Rats and treatment}

For the present experiment, twenty-nine GF male SpragueDawley rats bred in our animal facility from stock obtained from Charles River were used. Experimental conditions were as described previously ${ }^{(24)}$. Whereas seventeen rats were kept under GF conditions, twelve rats were associated at 10 weeks of age with human intestinal microbiota via intragastric application of a $1 \mathrm{ml}$ faecal suspension from a human donor $(1: 50, \mathrm{w} / \mathrm{v}$, in $0.85 \% \mathrm{NaCl}, \mathrm{w} / \mathrm{v})$ as described previously $^{(24)}$. The composition of the intestinal microbiota in HMA rats was stable throughout the study period $^{(24)}$, which corresponds to data reported in a previous long-term study with rats ${ }^{(25)}$. The microbiological status of GF rats was confirmed throughout the study as described previously ${ }^{(26)}$. The rats were fed a semi-purified flavonoid-free diet during the experiments $^{(24)}$. At 13 weeks of age, $1 \mathrm{ml}$ of $0 \cdot 1 \%$ aqueous citric acid (w/v) was intragastrically administered to each rat as a control. Urine and faeces were collected using metabolism cages (Tecniplast) for $24 \mathrm{~h}$ after control application and pooled for every $12 \mathrm{~h}$. C3G at a mean dose of 91.7 (SD $2 \cdot 3) \mu \mathrm{mol} / \mathrm{kg}$ body weight in $1 \mathrm{ml}$ of $0 \cdot 1 \%$ aqueous citric acid (w/v) was intragastrically applied twice to each rat, at 14 and 20 weeks of age. Following each C3G application, urine and faeces were collected for $48 \mathrm{~h}$, pooled for every $12 \mathrm{~h}$. The results obtained for the first and second C3G applications revealed no significant differences. Thus, the two datasets were combined for each rat. At the end of the study, the rats were killed by $\mathrm{CO}_{2}$ inhalation and pathohistological examinations of liver, lung, kidney, myocardium and spleen were conducted. Furthermore, differential blood counts were determined (Vet-Med-Labor). According to these examinations, weight gain and the overall behaviour, the rats' health status was not affected by bacterial colonisation or C3G application. The institutional and national guidelines for the care and use of animals were followed and all experimental procedures involving animals were approved by the Office for Agriculture, Ecology and Regional Planning of the State Brandenburg according to $\int$ 8.I Animal Welfare Act.

\section{Preparation of rat samples}

Urine was filtered sterile immediately after sampling and acidified with 0.2 volumes of TFA $(0.44 \mathrm{M})$. Until further processing, faeces and acidified urine were stored at $-80^{\circ} \mathrm{C}$. To extract $\mathrm{C} 3 \mathrm{G}$ and its metabolites from the urine, RPOasis-HLB cartridges $(30 \mathrm{mg}, 30 \mu \mathrm{m}$; Waters) were used, which had been conditioned by washing with $1 \mathrm{ml}$ methanol and equilibrated by washing with $1 \mathrm{ml}$ of water. After the application of the acidified urine sample $(1.2 \mathrm{ml})$, the cartridge was washed with $1 \mathrm{ml}$ of $1 \mathrm{~mm}$-aqueous $\mathrm{HCl}$ and $1 \mathrm{ml}$ of $1 \mathrm{~mm}-\mathrm{HCl}$ in $20 \%$ methanol. Elution was performed using 
$1 \mathrm{ml}$ of $1 \mathrm{~mm}-\mathrm{HCl}$ in $90 \%$ methanol. The eluates were dried by vacuum centrifugation (RC 10.22, Jouan) and dissolved in the original volume of methanol-water-TFA (90:9:1, by vol.). Faecal samples were thawed, acidified with $0 \cdot 25$ volumes ( $v / w$, relating to wet weight of the original faecal sample) of formic acid $(5 \mathrm{M})$ and lyophilised. Then, eight volumes $(\mathrm{v} / \mathrm{w})$ of methanol- $\mathrm{HCl}(99: 1, \mathrm{v} / \mathrm{v})$ were added, and the samples were sonicated for $30 \mathrm{~min}$ and centrifuged $(7500 \mathrm{~g})$ for $10 \mathrm{~min}$ at room temperature. Extraction was repeated five times. The pooled supernatants were dried by vacuum centrifugation. The extraction of urine and faeces was carried out in duplicate. Glucurono- and sulpho-conjugates were determined indirectly by comparative HPLC analysis of samples with and without enzymatic hydrolysis of the conjugated forms using extracts of Helix pomatia as described previously ${ }^{(24)}$.

To determine the recovery of $\mathrm{C} 3 \mathrm{G}$, one-fifth of the samples collected during the study were extracted and analysed a second time after spiking with $25 \mu \mathrm{M} \mathrm{C} 3 \mathrm{G}$. The C3G recovery was $41 \%$ for urine and $43 \%$ for faeces. In the preliminary experiments, the recovery of cyanidin and peonidin was observed to be comparable with that of C3G. Thus, the values obtained by HPLC analysis of the content of C3G, cyanidin and peonidin in the samples were corrected for the relative recovery of $\mathrm{C} 3 \mathrm{G}$. DHBA, THBA, VA and THBAld were recovered completely in the preliminary tests.

\section{HPLC analysis}

The dried samples were each dissolved in the original volume of methanol-water-TFA (90:9:1, by vol.), sonicated at $45 \mathrm{kHz}$ for 5 min (Ultrasonic bath Transonic $\mathrm{T} 480$; Elma) and centrifuged $(18200 \mathrm{~g}, 5 \mathrm{~min})$. Aliquots $(20 \mu \mathrm{l})$ of the supernatants were analysed by a Summit HPLC system (Dionex) consisting of a pump (P 680A LPG), an autosampler (ASI-100T), a column oven (TCC-100), a diode-array detector (UVD 340U PDA) and an RP-Zorbax SB-C18 column (5 $\mu \mathrm{m}$, $4.6 \times 150 \mathrm{~mm})$ equipped with a RP-SB-C18 guard column $(5 \mu \mathrm{m})$ (Agilent Technologies). The column temperature was maintained at $35^{\circ} \mathrm{C}$. The mobile phase was a mixture of $0 \cdot 1 \mathrm{~m}$-aqueous TFA ( $\mathrm{pH} \mathrm{1}$, solvent A), methanol (solvent B) and water (solvent $\mathrm{C}$ ) delivered at $1 \mathrm{ml} / \mathrm{min}$ with a linear gradient of $0-90 \% \mathrm{~B}$ in $25 \mathrm{~min}$, while solvent $\mathrm{C}$ decreased from 90 to $0 \%$. Detection was at 250, 285 and $520 \mathrm{~nm}$. Calibration curves of the corresponding standard compounds were used for quantification of the identified compounds. For unidentified compounds, peak areas were determined at the given wavelength.

\section{Ultra-performance liquid chromatography-electrospray ionisation-MS analysis}

An ultra-performance liquid chromatography (UPLC) system (Acquity Ultra Performance LC; Waters) consisting of a solvent manager, a sample manager and a diode-array detector was used and connected to a triple quadrupole mass spectrometer with Z-spray atmospheric pressure electrospray ionisation source (Quattro Premier XE; Waters). The column was a UPLC bridged ethyl hybrid (BEH) phenyl column $(1.7 \mu \mathrm{m}$,
$50 \mathrm{~mm} \times 2 \cdot 1 \mathrm{~mm}$; Waters). The column temperature was maintained at $25^{\circ} \mathrm{C}$. The solvents were water-formic acid (95:5, v/ $\mathrm{v}, \mathrm{pH} 1.7$, solvent A) and methanol (solvent B). They were delivered at $0.35 \mathrm{ml} / \mathrm{min}$ with a gradient of $0-10 \% \mathrm{~B}$ in $2.65 \mathrm{~min}$, hold at $10 \%$ for $1.46 \mathrm{~min}$ and from 10 to $100 \%$ in $4.39 \mathrm{~min}$. A $4 \mu \mathrm{l}$ aliquot of the samples was injected. MS analyses were carried out in positive ionisation mode at a capillary voltage of $0.7 \mathrm{kV}$, a source block temperature of $110^{\circ} \mathrm{C}$ and a desolvation temperature of $450^{\circ} \mathrm{C}$. The collision gas was $\mathrm{Ar}$ at a pressure of $0.30 \mathrm{~Pa}$. The cone voltage was varied between 20 and $60 \mathrm{~V}$ and the collision energy between 4 and $35 \mathrm{eV}$. Data were analysed using MassLynx Software version 4.1 (Waters).

\section{Data evaluation and statistical analysis of the animal study}

Statistical analysis was carried out using software SPSS 11.5 (SPSS, Inc.). Values were tested for normal distribution using the Shapiro-Wilk test. Time effects were tested by analysing differences between the two C3G applications in GF and HMA rats using a paired $t$ test or, when data were nonnormally distributed, by the Wilcoxon test. Independent data were tested for homogeneity of variance by the Levene test and differences were checked for significance using an unpaired $t$ test when data were normally distributed. Differences in non-normally distributed data were checked for significance by the Mann-Whitney test. Differences between the GF and HMA rats were considered significant at $P \leq 0 \cdot 05$. Data are presented as medians.

\section{Results}

\section{Stability of cyanidin 3-glucoside and cyanidin during simulated gastrointestinal passage}

The stability of $\mathrm{C} 3 \mathrm{G}$ and its aglycone cyanidin during the gastrointestinal passage was tested by incubation for $2 \mathrm{~h}$ with simulated gastric and intestinal fluids, respectively. The treatment with simulated gastric fluid decreased C3G and cyanidin concentrations to 75.3 (SD 5.0) and $48 \cdot 2$ (SD 9.1) \% of its initial value, respectively. DHBA and THBAld were identified as decomposition products of cyanidin. Incubation with simulated intestinal fluid resulted in a C3G and cyanidin recovery of $76.3(\mathrm{SD} 10.5)$ and 10.9 (SD 15.5) \%, respectively.

\section{Conversion of cyanidin 3-glucoside by human intestinal bacteria}

Faecal slurries prepared from each of the four human donors converted C3G within 2 h completely, while the C3G concentration remained widely stable in the absence of microbiota (90.6 (SD 14.7)\% after $24.5 \mathrm{~h}$ of incubation; Fig. 1(a)). DHBA, THBAld and four unknown products (P1, P2, P3 and P4) were detected during incubation with faecal slurries (Fig. 1(b)). Of the applied C3G (66.0 (SD 4.4) $\mu \mathrm{M})$, a maximum of 6.46 (SD 0.98) $\mu \mathrm{M}$ was recovered as DHBA and 1.38 (SD 0.83 ) $\mu \mathrm{M}$ as THBAld after $10 \mathrm{~h}$ of incubation. P1, P2, P3 and $\mathrm{P} 4$ were detected as from $30 \mathrm{~min}$ of incubation with 
human faecal slurries. The peak area of P1 was maximal at $3 \mathrm{~h}$ (area under the curve (AUC) $285 \mathrm{~nm}, 2.48$ (SD 0.90)). The peak areas of $\mathrm{P} 3$ plateaued after $3 \mathrm{~h}\left(\mathrm{AUC}_{285 \mathrm{~nm}}, 0 \cdot 16\right.$ (SD 0.07)), of $\mathrm{P} 4$ after $8 \mathrm{~h}\left(\mathrm{AUC}_{285 \mathrm{~nm}}, 0 \cdot 12\right.$ (SD 0.14)) and of P2 after $24 \cdot 5 \mathrm{~h}$ of incubation $\left(\mathrm{AUC}_{250 \mathrm{~nm}}, 1.41\right.$ (SD 0.80)). DHBA was also detected during the incubation of C3G (75.5 (SD 12.4) $\mu \mathrm{M})$ without faecal microbiota but at a much lower concentration $(0.88(\mathrm{SD} 0.18) \mu \mathrm{m})$ after $10 \mathrm{~h}$ of incubation compared with C3G incubation with faecal slurries (Fig. 1(c)). The incubation of the aglycone cyanidin $(61 \cdot 4(\mathrm{SD} 30 \cdot 1) \mu \mathrm{M})$ in the absence of bacteria yielded DHBA, THBAld, P1 and P2 but at clearly lower levels compared with the incubation of C3G with faecal slurries (Fig. 1(d)). The concentration of DHBA and THBAld at $10 \mathrm{~h}$ of incubation was 1.20 (SD 0.13 ) and 0.93 (SD 0.11) $\mu \mathrm{M}$, respectively. The maximum peak areas were detected for P1 after $3 \mathrm{~h}\left(\mathrm{AUC}_{285 \mathrm{~nm}}, 1.06\right.$ (SD 0.64)) and for $\mathrm{P} 2$ after $24 \mathrm{~h}$ of incubation $\left(\mathrm{AUC}_{250 \mathrm{~nm}}, 0.75\right.$ (SD 0.25)). Cyanidin was as stable as $\mathrm{C} 3 \mathrm{G}$ in the absence of faecal microbiota under the conditions used (Fig. 1(a)).

The ability of intestinal bacterial species to convert C3G was tested with $E$. ramulus and $C$. saccharogumia, which are known to degrade polyphenolic glycosides ${ }^{(27,28)}$. The incubation of $\mathrm{C} 3 \mathrm{G}$ for $48 \mathrm{~h}$ with $E$. ramulus and $C$. saccharogumia decreased its concentration by 79.7 (SD 2.1) and 92.7 (SD 3.5$) \%$, respectively. Within the same time period, the
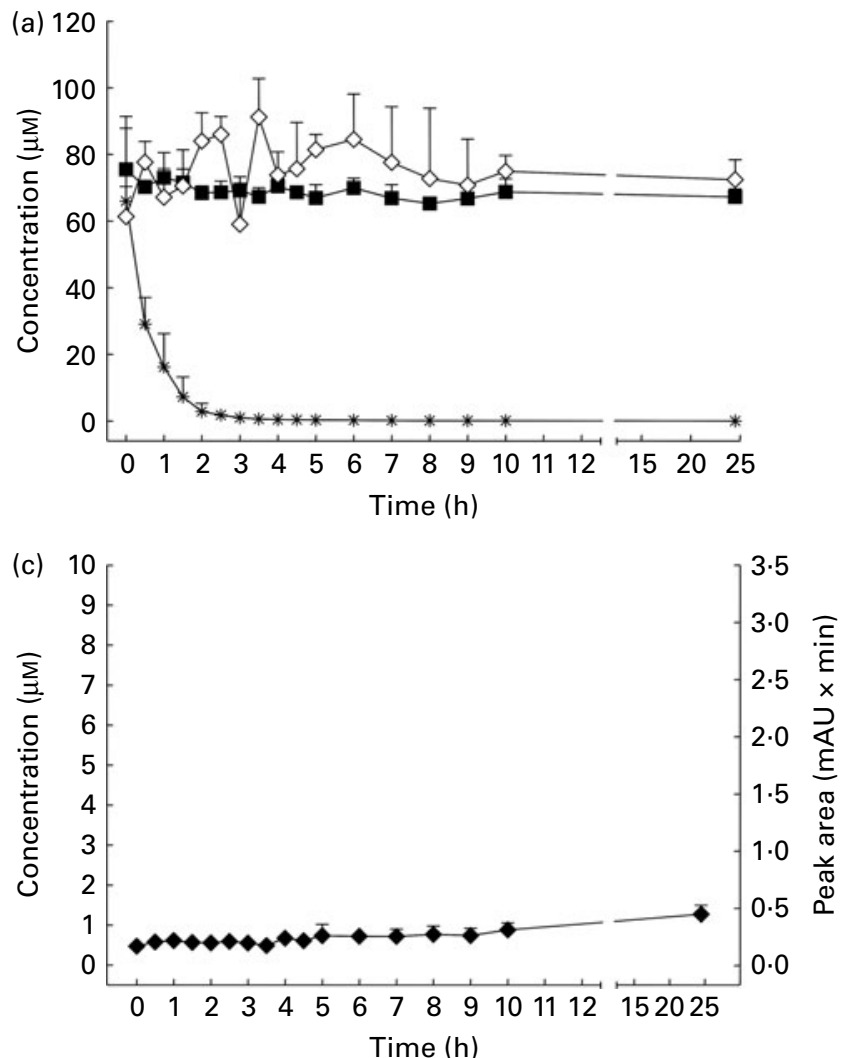

C3G concentration decreased by only 20.6 (SD 0.8 ) \% in the absence of bacteria. The incubation of C3G (50.0 (SD 12.9) $\mu \mathrm{M}$ ) with E. ramulus resulted in the formation of 8.65 (SD 1.20) $\mu \mathrm{m}$-DHBA, 1.82 (SD 0.34) $\mu \mathrm{m}$-THBAld, P1 $\left(\mathrm{AUC}_{285 \mathrm{~nm}}, 0 \cdot 17(\mathrm{SD} 0 \cdot 15)\right)$ and P2 (AUC $250 \mathrm{~nm}, 1 \cdot 11$ (SD 0.35)) within $48 \mathrm{~h}$. The same pattern of the products at similar concentrations was observed when C3G (51.7 (SD 5.6) $\mu \mathrm{M})$ was incubated with C. saccharogumia for $48 \mathrm{~h}: 5.59$ (SD 3.00) $\mu \mathrm{M}-$ DHBA, 1.36 (SD 0.88) $\mu \mathrm{m}$-THBAld, P1 (AUC $285 \mathrm{~nm}, \quad 0.56$ (SD 0.27)) and P2 (AUC $250 \mathrm{~nm}, 0.83(\mathrm{SD} 0 \cdot 27)$ ). Only DHBA at a lower concentration $(3.42(\mathrm{SD} 0 \cdot 28) \mu \mathrm{M})$ was formed spontaneously from C3G (65.6 (SD 1.2) $\mu \mathrm{m})$ within $48 \mathrm{~h}$.

The results indicate that $\mathrm{C} 3 \mathrm{G}$ was degraded rapidly by bacteria via cyanidin to DHBA, THBAld, P1 and P2. These products could possibly also have been formed spontaneously from cyanidin, in particular at neutral $\mathrm{pH}$ inside the bacterial cells. In contrast, P3 and P4 were formed only in the presence of faecal microbiota but not by E. ramulus or C. saccharogumia.

\section{Characterisation of the products formed during bacterial cyanidin 3-glucoside conversion}

The spectroscopic properties of the products P1 $\left(\lambda_{\max }=291 /\right.$ $319 \mathrm{~nm}), \mathrm{P} 2\left(\lambda_{\max }=316 \mathrm{~nm}\right), \mathrm{P} 3\left(\lambda_{\max }=282 / 317 \mathrm{~nm}\right)$ and $\mathrm{P} 4$
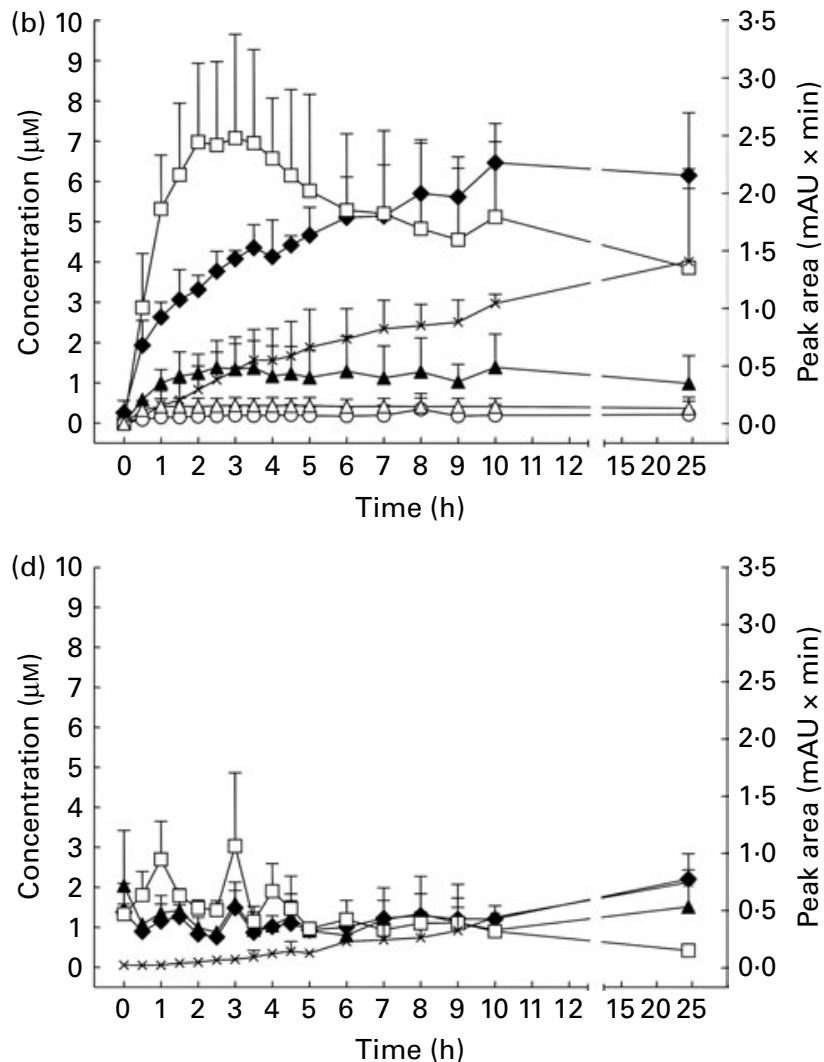

Fig. 1. (a) Incubation of cyanidin 3-glucoside (C3G) with human faecal slurries $(*)$, and control incubations of $C 3 G(\square)$ and cyanidin ( $\diamond)$ without an inoculum. Formation of the products during incubations shown under (a): (b) C3G with human faecal slurries, (c) C3G without an inoculum and (d) cyanidin without an inoculum. C3G, cyanidin, 3,4-dihydroxybenzoic acid ( $)$ and 2,4,6-trihydroxybenzaldehyde $(\boldsymbol{\Delta})$ were quantified by HPLC-diode-array detector based on calibration curves of the corresponding standard compounds. Peak areas were determined for P1 ( $\square$ ), P3 ( $\triangle$ ) and P4 (O) at $285 \mathrm{~nm}$ and for P2 ( $\times)$ at $250 \mathrm{~nm}$ ( $y$-axis on the right). Values are means of faecal slurries of the four tested human donors and, for controls, of three repetitions, with standard deviations represented by vertical bars. AU, arbitrary units. 
$\left(\lambda_{\max }=282 / 317 \mathrm{~nm}\right)$ differed from those of both C3G $\left(\lambda_{\max }=280 / 518 \mathrm{~nm}\right)$ and cyanidin $\left(\lambda_{\max }=276 / 530 \mathrm{~nm}\right)$. In the HPLC analysis, P1 $\left(R_{\mathrm{t}}=9.9 \mathrm{~min}\right)$ and $\mathrm{P} 2\left(R_{\mathrm{t}}=11.7 \mathrm{~min}\right)$ eluted earlier than cyanidin $\left(R_{\mathrm{t}}=14.5 \mathrm{~min}\right)$. In addition to their similar UV spectra, P3 and $\mathrm{P} 4$ had similar retention times of $16 \cdot 8$ and $16.5 \mathrm{~min}$, respectively.

The four products were further characterised by the electrospray ionisation-MS analysis. The protonated molecule of P1 was most probably represented by $m / z 305[\mathrm{M}+\mathrm{H}]^{+}$, which was supported by further signals at $m / z 327\left[\mathrm{M}+\mathrm{Na}^{+}\right.$and $m / z 343[\mathrm{M}+\mathrm{K}]^{+}$. The optimised product-ion spectrum of $\mathrm{m} / z 305$ revealed a base peak at $\mathrm{m} / z 137$ consistent with the B-ring-derived dihydroxybenzoyl cation, while other fragment ions showed less than $8 \%$ base peak intensity (Fig. 2). Anthocyanins are known to form chalcones at $\mathrm{pH}$ $>7^{(29,30)}$. Based on its maximum UV absorption at $320 \mathrm{~nm}$, which is typical of chalcones ${ }^{(30)}$, and its molecular mass of $304, \mathrm{P} 1$ is proposed to represent the cyanidin-derived $\alpha$-hydroxychalcone.

The MS spectrum of P2 with signals at $m / z 301[\mathrm{M}+\mathrm{H}]^{+}$, $m / z 323[\mathrm{M}+\mathrm{Na}]^{+}$and $m / z 339[\mathrm{M}+\mathrm{K}]^{+}$indicates a molecular mass of 300 . Owing to the limited amount of $\mathrm{P} 2$, a production spectrum could not be recorded. P2 may result from the oxidation of $\mathrm{P} 1$, with the A-ring forming a quinone methide and the B-ring an 1,2-quinone (for ring designation, see Fig. 6).

The MS analysis of $\mathrm{P} 3$ and $\mathrm{P} 4$ resulted in complex spectra with postulated protonated molecules $[\mathrm{M}+\mathrm{H}]^{+}$at $\mathrm{m} / z 607$ and $m / z 605$, respectively, and $[\mathrm{M}+\mathrm{Na}]^{+}$signals at $m / z 629$ and $m / z 627$, respectively. Fragmentation of the protonated molecules of $\mathrm{P} 3$ and $\mathrm{P} 4$ revealed a pattern of signals similar to each other (Fig. 3(a) and (b)). The difference of two mass units between the molecular peaks and individual fragment ions of $\mathrm{P} 3$ and $\mathrm{P} 4(\mathrm{~m} / z 589 v .587, \mathrm{~m} / z 346 v$. 344) indicates that the two compounds differ in their redox state. However, other $\mathrm{P} 3$ and $\mathrm{P} 4$ specific fragments varied by only one mass unit $(m / z 482 v$. 481, $m / z 464 v$. 463). Both the retention times and the spectroscopic behaviour support the close structural similarity of $\mathrm{P} 3$ and $\mathrm{P} 4$. P3 may be the product of either homodimerisation of P1 followed by the oxidation of one of the four aromatic rings or by heterodimerisation of P1 and an oxidised P1 molecule. P4 could subsequently have

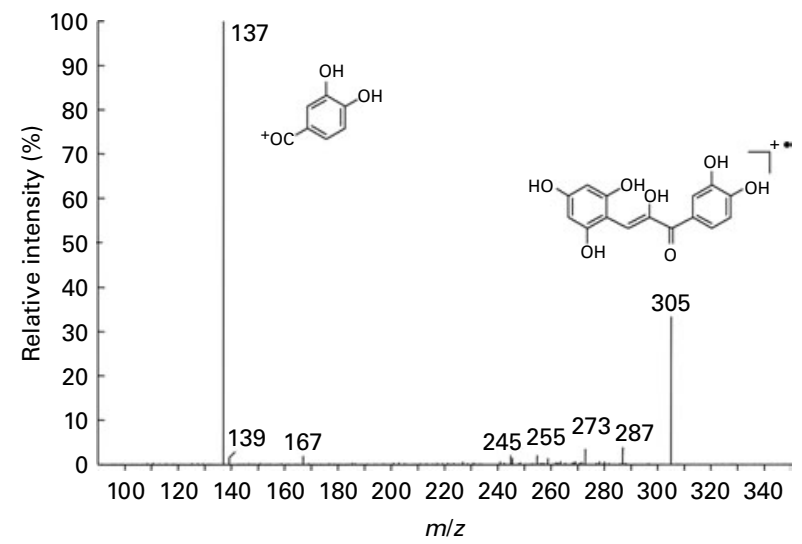

Fig. 2. Product-ion spectrum of the protonated molecule of the product $P 1$ at $\mathrm{m} / \mathrm{z} 305[\mathrm{M}+\mathrm{H}]^{+}$obtained at a cone voltage of $20 \mathrm{~V}$ and a collision energy of $10 \mathrm{eV}$.
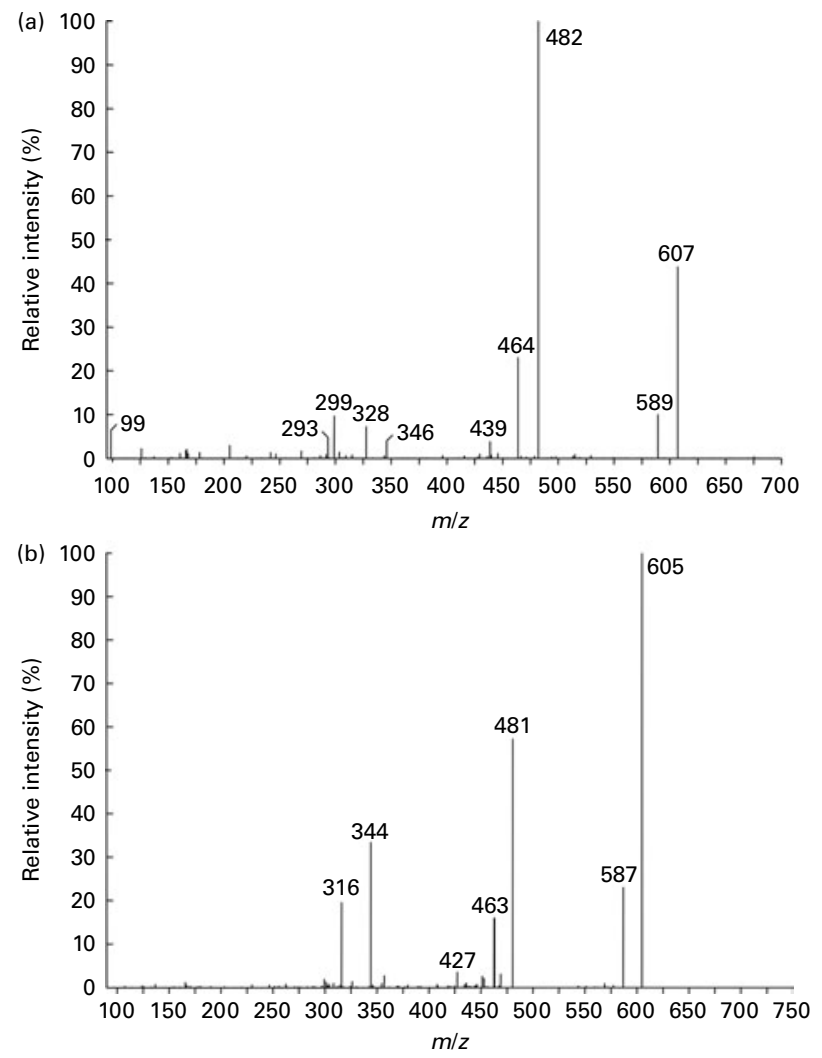

Fig. 3. Product-ion spectra of the protonated molecules of (a) the product P3 at $m / z 607[\mathrm{M}+\mathrm{H}]^{+}$and (b) the product $\mathrm{P} 4$ at $\mathrm{m} / \mathrm{z} 605[\mathrm{M}+\mathrm{H}]^{+}$obtained at a cone voltage of $35 \mathrm{~V}$ and a collision energy of $25 \mathrm{eV}$.

been formed by further oxidation of P3. A product of spontaneous cyanidin decomposition with a protonated molecule at $m / z 605[\mathrm{M}+\mathrm{H}]^{+}$was previously proposed to be a dimerisation product of two cyanidin units ${ }^{(15)}$.

\section{Metabolism of cyanidin 3-glucoside in germ-free and human microbiota-associated rats}

The GF and HMA rats excreted C3G, cyanidin, DHBA, THBAld and VA with urine after the oral application of C3G. All compounds also occurred in their conjugated forms except for C3G and cyanidin in HMA rats (Fig. 4). DHBA and VA were the main metabolites in both GF and HMA rats; however, the HMA rats excreted lower proportions as free DHBA and conjugated VA than the GF rats (Fig. 4). Peonidin and HCA in their free and conjugated forms occurred exclusively in the urine of HMA rats but not of GF rats.

The GF and HMA rats excreted C3G, cyanidin, DHBA, THBAld and THBA in their free and conjugated forms with faeces throughout the observation period of $48 \mathrm{~h}$ after $\mathrm{C} 3 \mathrm{G}$ application (Fig. 5). The HMA rats excreted a higher proportion of $\mathrm{C} 3 \mathrm{G}$ as free DHBA, conjugated THBAld and THBA in its free and conjugated forms with faeces than the GF rats.

The total excretion of $\mathrm{C} 3 \mathrm{G}$ and its metabolites within $48 \mathrm{~h}$ after C3G application via the urine and faeces was low in both HMA and GF rats, accounting for 3.7 and $1.7 \%$, respectively, of the ingested C3G dose. The time course of excretion 
of the identical compounds did not differ between HMA and GF rats. The main excretion route was faeces with $87.8 \%$ of total $\mathrm{C} 3 \mathrm{G}$ excreted in HMA rats and $68.9 \%$ in GF rats, with compounds in their free form largely dominating. The HMA rats excreted more free compounds and conjugates with faeces than the GF rats (Fig. 5), while the opposite was true for the excretion of conjugates with urine (Fig. 4).

Several unknown metabolites were detected after the application of $\mathrm{C} 3 \mathrm{G}$ in the urine and faeces of HMA rats, which were not present in the samples of GF rats. These compounds could not be assigned to either the metabolites P1 to P4 observed in the course of in vitro incubation of $\mathrm{C} 3 \mathrm{G}$ with human faecal slurries or the following authentic standards: ferulic acid; 3,4-dihydroxycinnamic acid; 3-(3-hydroxyphenyl)propionic acid; 3-phenylpropionic acid; 3-hydroxyphenylacetic acid; phenylacetic acid; 3-methoxybenzoic acid; 2,4-dihydroxybenzoic acid; 2,6-dihydroxybenzoic acid; 2-hydroxybenzoic acid; 3-hydroxybenzoic acid; 4-hydroxybenzoic acid; benzoic acid; catechol; hippuric acid.

\section{Discussion}

Compared with other flavonoids, the bioavailability of anthocyanins is very low. Maximum plasma levels of total anthocyanins are in the range of $1-100 \mathrm{~nm}$ with doses of $7-1618 \mu \mathrm{mol}$ in human studies ${ }^{(31)}$. Typically less than $1 \%$ of the ingested dose are excreted in the urine by laboratory animals ${ }^{(10)}$, while higher recoveries of up to $5 \%$ have been reported after the intake of anthocyanin-rich foods by human subjects ${ }^{(32)}$. However, 60-90\% of the anthocyanins were demonstrated to disappear from the gastrointestinal tract within $4 \mathrm{~h}$ after a meal, and it has been hypothesised that degradation accounts for this disappearance ${ }^{(31)}$. Anthocyanins are in general stable

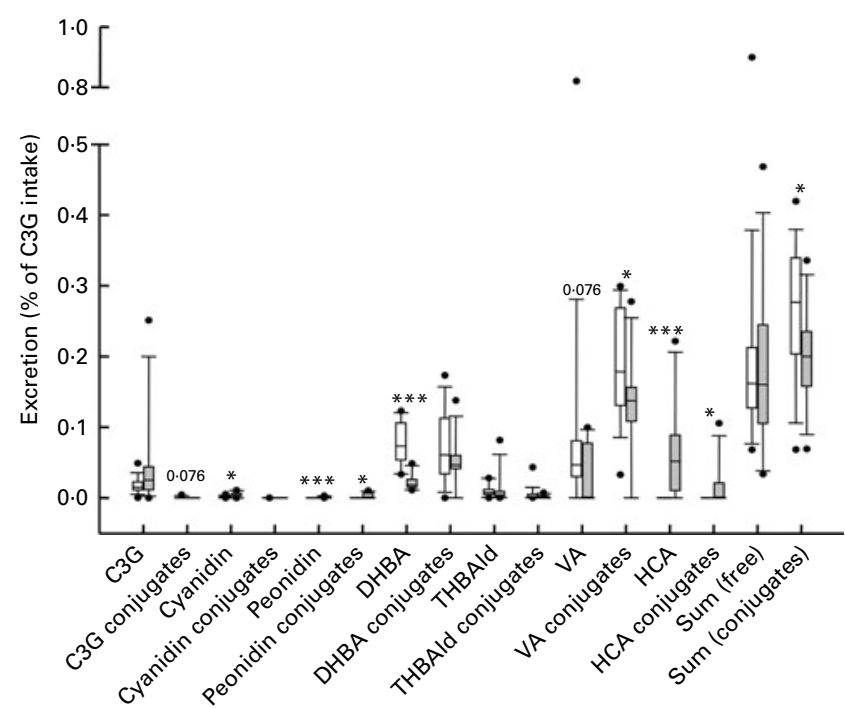

Fig. 4. Excretion of cyanidin 3-glucoside (C3G) and its metabolites via the urine of germ-free (GF) rats $(n 17, \square)$ and human microbiota-associated (HMA) rats $(n 12, \square)$ within $48 \mathrm{~h}$ after the application of C3G. Depicted are median (inner line), 25-75 percentile (box), 10-90 percentile (stamp) and outliers (•). Values were significantly different between GF and HMA rats: ${ }^{*} P \leq 0.05,{ }^{* * *} P \leq 0.001$. DHBA, 3,4-dihydroxybenzoic acid; HCA, 3-hydroxycinnamic acid; THBAld, 2,4,6-trihydroxybenzaldehyde; VA, vanillic acid.

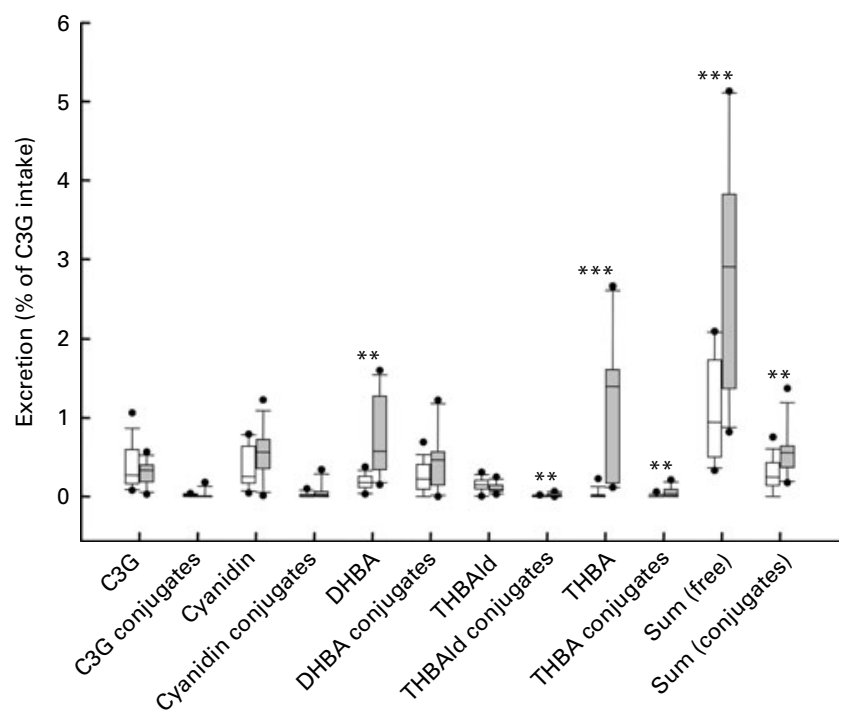

Fig. 5. Excretion of cyanidin 3-glucoside (C3G) and its metabolites via the faeces of germ-free (GF) rats $(n 17, \square)$ and human microbiota-associated (HMA) rats $(n 12, \square)$ within $48 \mathrm{~h}$ after the application of C3G. Depicted are median (inner line), 25-75 percentile (box), 10-90 percentile (stamp) and outliers ( $(\bullet)$. Values were significantly different between GF and HMA rats: ${ }^{\star *} P \leq 0.01,{ }^{* \star *} P \leq 0.001$. DHBA, 3,4-dihydroxybenzoic acid; THBAld, 2,4,6-trihydroxybenzaldehyde; THBA, 2,4,6-trihydroxybenzoic acid.

during incubation with simulated gastric and intestinal fluids ${ }^{(33-36)}$. This was also true for $\mathrm{C} 3 \mathrm{G}$ in our hands, whereas its aglycone cyanidin was less stable, resulting in the formation of DHBA and THBAld. The in vitro incubation data and results from studies with ileostomists ${ }^{(12)}$ indicate that significant amounts of dietary anthocyanins reach the large intestine where bacterial degradation may occur. In addition, absorbed anthocyanins are also excreted with bile into the colon ${ }^{(37)}$. Since the contribution of intestinal bacteria to the metabolism of anthocyanins in vivo is still not known, we aimed to elucidate the impact of human gut microbiota on the fate of $\mathrm{C} 3 \mathrm{G}$ by comparing the metabolism of $\mathrm{C} 3 \mathrm{G}$ in HMA and GF rats. Although urine and faeces were analysed for all the known degradation products derived from $\mathrm{C} 3 \mathrm{G}$ so far and other potential metabolites, the total recovery of the applied C3G dose within $48 \mathrm{~h}$ was only $3.7 \%$ for HMA rats. The minor proportion of $0.4 \%$ was excreted with urine, which is in the same range as reported for $\mathrm{C} 3 \mathrm{G}$ in previous studies with rodents or human subjects ${ }^{(38-46)}$. When radiolabelled C3G was applied to mice, $3.3 \%$ of radioactivity appeared in the urine while $44.5 \%$ were recovered in the faeces collected for $24 \mathrm{~h}$ after C3G ingestion ${ }^{(47)}$. We recovered only $3.3 \%$ of the applied $\mathrm{C} 3 \mathrm{G}$ dose in the form of $\mathrm{C} 3 \mathrm{G}$ and identified products in the faeces of HMA rats within $48 \mathrm{~h}$. A recovery of $72 \%$ of the ingested $\mathrm{C} 3 \mathrm{G}$ in the form of DHBA in the blood (44\%) and faeces (28\%) has been reported in one human study following the ingestion of blood orange juice ${ }^{(19)}$. However, it cannot be excluded that DHBA detected in this study was formed from polyphenolic compounds other than $\mathrm{C} 3 \mathrm{G}$ contained in the blood orange juice. In the present rat study with pure $\mathrm{C} 3 \mathrm{G}$, only $1.2 \%$ of the dose were recovered as DHBA in the urine and faeces of HMA rats. 


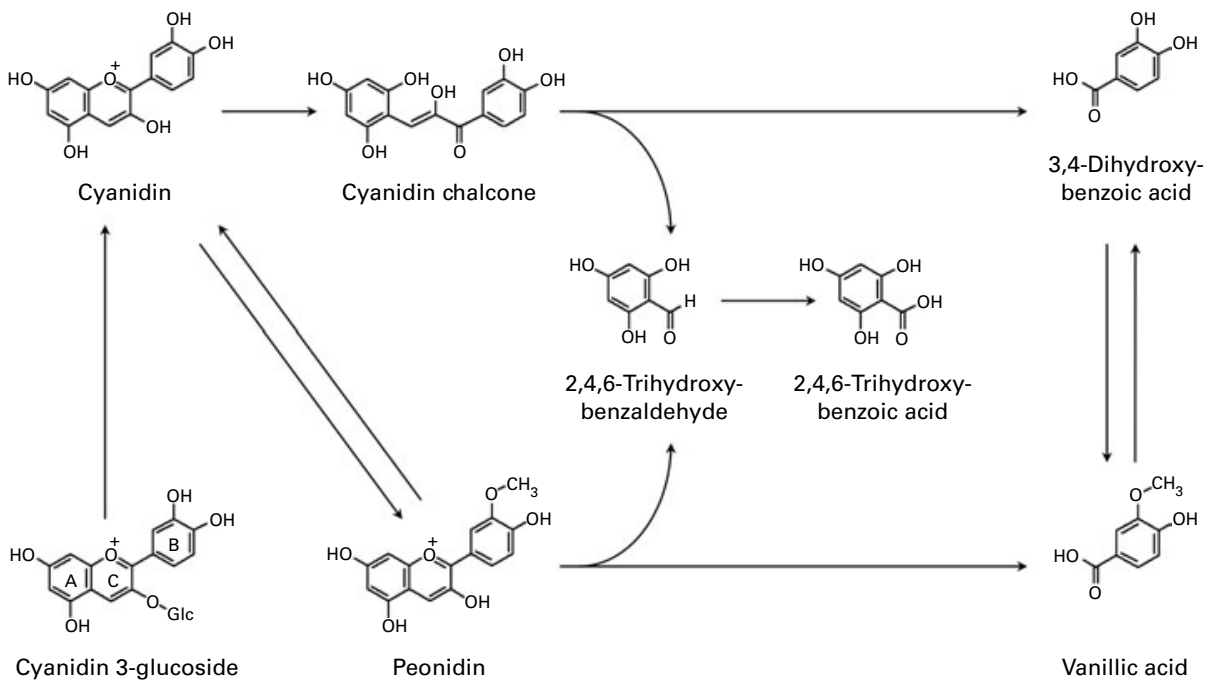

Fig. 6. Postulated metabolism of cyanidin 3-glucoside in human microbiota-associated rats. The cyanidin chalcone (P1) was only detected in vitro. Glc, glucosyl moiety.

Interestingly, the recovery of $\mathrm{C} 3 \mathrm{G}$ was even low in GF rats corresponding to only $1.7 \%$ of the applied dose. Thus, the degradation of $\mathrm{C} 3 \mathrm{G}$ by the intestinal microbiota is not the main reason for the low recovery observed in the present and previous studies. The excretion kinetics did not indicate an accumulation of $\mathrm{C} 3 \mathrm{G}$ and its products in tissues either in GF or HMA rats. Accumulation of compounds was also excluded in a previous mouse study using radiolabelled $\mathrm{C} 3 \mathrm{G}^{(47)}$. In the present study, several other compounds in addition to DHBA were observed after C3G application in both GF and HMA rats, and further products may have escaped detection. However, the increased faecal levels of DHBA, THBAld and THBA in HMA rats compared with GF rats led to an improved recovery of the $\mathrm{C} 3 \mathrm{G}$ dose. This finding and the appearance of peonidin and HCA exclusively in the urine of HMA rats indicate an influence of the intestinal microbiota on the metabolism of C3G in the host.

The present in vitro studies have also shown the accelerated formation of DHBA and THBAld from C3G in the presence of human intestinal bacteria. It may be concluded that the bacteria catalysed the deglycosylation of C3G, which subsequently led to the increased formation of DHBA and THBAld by bacterial and/or spontaneous degradation of cyanidin. The slightly increased urinary levels of cyanidin in HMA rats compared with GF rats and the occurrence of peonidin, the product of cyanidin methylation, exclusively in the urine of HMA rats corroborate this assumption. Cyanidin and its conjugates detected in GF rats were most probably formed by spontaneous decomposition since mammalian glycosidases appear not to act on $\mathrm{C} 3 \mathrm{G}^{(48,49)}$. Thus, the enzymatic deglycosylation of $\mathrm{C} 3 \mathrm{G}$ is catalysed by intestinal bacteria including E. ramulus and $C$. saccharogumia. The resulting cyanidin is further degraded to DHBA, THBAld and other products depending on the conditions. DHBA and THBAld have been detected after in vitro incubation of $\mathrm{C} 3 \mathrm{G}$ with faecal suspensions from human subjects, rats and pigs in previous studies ${ }^{(13-17)}$. However, they are also formed through spontaneous decomposition of $\mathrm{C} 3 \mathrm{G}$ and cyanidin in the absence of bacteria ${ }^{(14,15,49)}$. This finding is also supported by the present in vitro experiments. The amounts of DHBA and THBAld detected even in vitro were much lower than expected from the disappearance of the parent C3G. Moreover, THBAld appeared at lower levels than DHBA in vitro and in vivo, which has been reported previously and is explained by the increased reactivity of aldehydes ${ }^{(49)}$. Following the incubation of C3G with intestinal bacteria, we observed several other products in addition to DHBA and THBAld. For instance, one compound (P1) was tentatively identified as cyanidin chalcone and was also formed spontaneously from cyanidin. Moreover, two other products (P3 and $\mathrm{P} 4$ ) were only formed in the presence of faecal microbiota and appeared to be dimers of the cyanidin chalcone. It is well known that anthocyanidins are stable at $\mathrm{pH} 1-3$ in the form of the flavylium cation but at $\mathrm{pH}>4$, the carbinol pseudobase, the chalcone pseudobase and the quinoidal base are formed ${ }^{(2)}$, which may dimerise and/or decompose to the corresponding phenolic acids and aldehydes ${ }^{(15)}$.

The present results suggest that the formation of $\mathrm{C} 3 \mathrm{G}$ products in HMA rats resulted from a combination of conversion steps catalysed by bacterial and host enzymes and spontaneous decomposition (Fig. 6). The methylation of cyanidin to peonidin and the oxidation of THBAld to THBA were only observed in rats but not during fermentation with intestinal bacteria, indicating catalysis of these reactions by host enzymes. VA may result from either the decomposition of peonidin ${ }^{(14)}$ or the methylation of DHBA by the host's catechol-O-methyltransferase ${ }^{(50)}$. On the other hand, the intestinal microbiota is able to demethylate peonidin and $\mathrm{VA}^{(14,51)}$. The mechanism of HCA formation in HMA rats remains to be clarified. Since HCA was not observed as a metabolite of intestinal bacteria in vitro, it is probably formed by the combined activities of bacterial and host enzymes.

In conclusion, phenolic acids, such as DHBA and THBA, formed increasingly in the presence of intestinal bacteria may contribute to the proposed health effects of C3G either directly in the gut or after their absorption from the colon, rather than the parent compound or its unstable derivatives. 
However, the fate of the majority of the ingested C3G has yet to be explored in future studies. Only the complete knowledge on the absorption and metabolism of $\mathrm{C} 3 \mathrm{G}$ and other anthocyanins will allow the elucidation of the mechanism of action of these bioactives.

\section{Acknowledgements}

This study was supported by the Deutsche Forschungsgemeinschaft (grant no. BR 2269/2). The authors would like to acknowledge Peter Winterhalter (Technical University of Braunschweig, Institute of Food Chemistry, Germany) for kindly providing C3G. The authors thank Ines Grüner, Ute Lehmann, Janet Krause and Renate Herzog for the care of the animals, and Sabine Schmidt, Anke Gühler and Elisabeth Meyer for technical assistance. L. H., G. L., S. S., M. B. and A. B. designed the research; L. H., W. E., G. L. and S. S. conducted the research; L. H., W. E., M. B. and A. B. analysed the data; L. H., M. B. and A. B. wrote the manuscript. A. B. had primary responsibility for the final content. All authors read and approved the final manuscript. The authors do not have any conflict of interest.

\section{References}

1. Wu X, Beecher GR, Holden JM, et al. (2006) Concentrations of anthocyanins in common foods in the United States and estimation of normal consumption. I Agric Food Chem 54, 4069-4075.

2. Clifford MN (2000) Anthocyanins-nature, occurrence and dietary burden. J Sci Food Agric 80, 1063-1072.

3. Mink PJ, Scrafford CG, Barraj LM, et al. (2007) Flavonoid intake and cardiovascular disease mortality: a prospective study in postmenopausal women. Am J Clin Nutr 85, 895-909.

4. Wang LS \& Stoner GD (2008) Anthocyanins and their role in cancer prevention. Cancer Lett 269, 281-290.

5. Hou DX, Fujii M, Terahara N, et al. (2004) Molecular mechanisms behind the chemopreventive effects of anthocyanidins. J Biomed Biotechnol 2004, 321-325.

6. Shukitt-Hale B, Lau FC \& Joseph JA (2008) Berry fruit supplementation and the aging brain. J Agric Food Chem 56, 636-641.

7. Heinonen M (2007) Antioxidant activity and antimicrobial effect of berry phenolics - a Finnish perspective. Mol Nutr Food Res 51, 684-691.

8. Watzl B, Briviba K \& Rechkemmer G (2002) Anthocyane. Ernährungsumschau 49, 148-150.

9. Prior RL (2004) Absorption and metabolism of anthocyanins: potential health effects. In Phytochemicals: Mechanism of Action, pp. 1-19 [MS Meskin, editor]. Boca Raton, FL: CRC Press.

10. McGhie TK \& Walton MC (2007) The bioavailability and absorption of anthocyanins: towards a better understanding. Mol Nutr Food Res 51, 702-713.

11. Williamson G \& Clifford MN (2010) Colonic metabolites of berry polyphenols: the missing link to biological activity? Br J Nutr 104, Suppl. 3, S48-S66.

12. Kahle K, Kraus M, Scheppach W, et al. (2006) Studies on apple and blueberry fruit constituents: do the polyphenols reach the colon after ingestion? Mol Nutr Food Res 50 , 418-423.
13. Aura AM, Martin-Lopez P, O'Leary KA, et al. (2005) In vitro metabolism of anthocyanins by human gut microflora. Eur J Nutr 44, 133-142.

14. Keppler K \& Humpf HU (2005) Metabolism of anthocyanins and their phenolic degradation products by the intestinal microflora. Bioorg Med Chem 13, 5195-5205.

15. Fleschhut J, Kratzer F, Rechkemmer G, et al. (2006) Stability and biotransformation of various dietary anthocyanins in vitro. Eur J Nutr 45, 7-18.

16. Hassimotto NM, Genovese MI \& Lajolo FM (2008) Absorption and metabolism of cyanidin-3-glucoside and cyanidin-3-rutinoside extracted from wild mulberry (Morus nigra L.) in rats. Nutr Res 28, 198-207.

17. Han SJ, Ryu SN, Trinh HT, et al. (2009) Metabolism of cyanidin-3-O- $\beta$-d-glucoside isolated from black colored rice and its antiscratching behavioral effect in mice. $J$ Food Sci 74, $\mathrm{H} 253-\mathrm{H} 258$.

18. Del Rio D, Borges G \& Crozier A (2010) Berry flavonoids and phenolics: bioavailability and evidence of protective effects. Br J Nutr 104, Suppl. 3, S67-S90.

19. Vitaglione P, Donnarumma G, Napolitano A, et al. (2007) Protocatechuic acid is the major human metabolite of cyanidin-glucosides. J Nutr 137, 2043-2048

20. Galvano F, Vitaglione P, Li Volti G, et al. (2008) Protocatechuic acid: the missing human cyanidins' metabolite. Mol Nutr Food Res 52, 386-387.

21. Tsuda T, Horio F \& Osawa T (1999) Absorption and metabolism of cyanidin 3-O- $\beta$-d-glucoside in rats. FEBS Lett 449, 179-182.

22. Schwarz M, Hillebrand S, Habben S, et al. (2003) Application of high-speed countercurrent chromatography to the largescale isolation of anthocyanins. Biochem Eng J 14, 179-189.

23. Braune A, Gütschow M, Engst W, et al. (2001) Degradation of quercetin and luteolin by Eubacterium ramulus. Appl Environ Microbiol 67, 5558-5567.

24. Hanske L, Loh G, Sczesny S, et al. (2009) The bioavailability of apigenin-7-glucoside is influenced by human intestinal microbiota in rats. J Nutr 139, 1095-1102.

25. Alpert C, Sczesny S, Gruhl B, et al. (2008) Long-term stability of the human gut microbiota in two different rat strains. Curr Issues Mol Biol 10, 17-24.

26. Kamlage B, Hartmann L, Gruhl B, et al. (1999) Intestinal microorganisms do not supply associated gnotobiotic rats with conjugated linoleic acid. I Nutr 129, 2212-2217.

27. Schneider H \& Blaut M (2000) Anaerobic degradation of flavonoids by Eubacterium ramulus. Arch Microbiol 173, $71-75$.

28. Clavel T, Lippman R, Gavini F, et al. (2007) Clostridium saccharogumia sp. nov. and Lactonifactor longoviformis gen. nov. sp. nov., two novel human faecal bacteria involved in the conversion of the dietary phytoestrogen secoisolariciresinol diglucoside. Syst Appl Microbiol 30, 16-26.

29. Preston NW \& Timberlake CF (1981) Separation of anthocyanin chalcones by high-performance liquid chromatography. J Chromatogr A 214, 222-228.

30. Furtado P, Figueiredo P, Dasneves HC, et al. (1993) Photochemical and thermal degradation of anthocyanidins. J Photochem Photobiol A Chem 75, 113-118.

31. Prior RL \& Wu X (2006) Anthocyanins: structural characteristics that result in unique metabolic patterns and biological activities. Free Radic Res 40, 1014-1028.

32. Lapidot T, Harel S, Granit R, et al. (1998) Bioavailability of red wine anthocyanins as detected in human urine. J Agric Food Chem 46, 4297-4302. 
33. McDougall GJ, Fyffe S, Dobson P, et al. (2005) Anthocyanins from red wine - their stability under simulated gastrointestinal digestion. Phytochemistry 66, 2540-2548.

34. Perez-Vicente A, Gil-Izquierdo A \& Garcia-Viguera C (2002) In vitro gastrointestinal digestion study of pomegranate juice phenolic compounds, anthocyanins, and vitamin C. J Agric Food Chem 50, 2308-2312.

35. Bermúdez-Soto MJ, Tomás-Barberán FA \& García-Conesa MT (2007) Stability of polyphenols in chokeberry (Aronia melanocarpa) subjected to in vitro gastric and pancreatic digestion. Food Chem 102, 865-874.

36. He J, Wallace TC, Keatley KE, et al. (2009) Stability of black raspberry anthocyanins in the digestive tract lumen and transport efficiency into gastric and small intestinal tissues in the rat. J Agric Food Chem 57, 3141-3148.

37. Talavera S, Felgines C, Texier O, et al. (2003) Anthocyanins are efficiently absorbed from the stomach in anesthetized rats. J Nutr 133, 4178-4182.

38. Borges G, Roowi S, Rouanet JM, et al. (2007) The bioavailability of raspberry anthocyanins and ellagitannins in rats. Mol Nutr Food Res 51, 714-725.

39. Felgines C, Talavera S, Texier O, et al. (2006) Absorption and metabolism of red orange juice anthocyanins in rats. Br J Nutr 95, 898-904.

40. McGhie TK, Ainge GD, Barnett LE, et al. (2003) Anthocyanin glycosides from berry fruit are absorbed and excreted unmetabolized by both humans and rats. J Agric Food Chem 51, 4539-4548.

41. Bitsch R, Netzel M, Sonntag S, et al. (2004) Urinary excretion of cyanidin glucosides and glucuronides in healthy humans after elderberry juice ingestion. J Biomed Biotechnol 2004, $343-345$.
42. Felgines C, Texier O, Besson C, et al. (2002) Blackberry anthocyanins are slightly bioavailable in rats. J Nutr $\mathbf{1 3 2}$, 1249-1253.

43. Felgines C, Talavera S, Texier O, et al. (2005) Blackberry anthocyanins are mainly recovered from urine as methylated and glucuronidated conjugates in humans. J Agric Food Chem 53, 7721-7727.

44. Marczylo TH, Cooke D, Brown K, et al. (2009) Pharmacokinetics and metabolism of the putative cancer chemopreventive agent cyanidin-3-glucoside in mice. Cancer Chemother Pharmacol 64, 1261-1268.

45. Felgines C, Texier O, Besson C, et al. (2008) Influence of glucose on cyanidin 3-glucoside absorption in rats. $\mathrm{Mol} \mathrm{Nutr}$ Food Res 52, 959-964.

46. Murkovic M, Mulleder U, Adam U, et al. (2001) Detection of anthocyanins from elderberry juice in human urine. $J$ Sci Food Agric 81, 934-937.

47. Felgines C, Krisa S, Mauray A, et al. (2010) Radiolabelled cyanidin 3-O-glucoside is poorly absorbed in the mouse. Br J Nutr 103, 1738-1745.

48. Nemeth K, Plumb GW, Berrin JG, et al. (2003) Deglycosylation by small intestinal epithelial cell $\beta$-glucosidases is a critical step in the absorption and metabolism of dietary flavonoid glycosides in humans. Eur J Nutr 42, 29-42.

49. Kay CD, Kroon PA \& Cassidy A (2009) The bioactivity of dietary anthocyanins is likely to be mediated by their degradation products. Mol Nutr Food Res 53, Suppl. 1, S92-S101.

50. Cao YG, Zhang L, Ma C, et al. (2009) Metabolism of protocatechuic acid influences fatty acid oxidation in rat heart: new anti-angina mechanism implication. Biochem Pharmacol 77, 1096-1104.

51. Scheline RR (1966) Decarboxylation and demethylation of some phenolic benzoic acid derivatives by rat caecal contents. J Pharm Pharmacol 18, 664-669. 